\title{
Comparative Effects of Triflusal, S-Adenosylmethionine, and Dextromethorphan over Intestinal Ischemia/Reperfusion Injury
}

\author{
Carlos R. Cámara-Lemarroy, ${ }^{1,2}$ Francisco J. Guzmán-de la Garza, ${ }^{1}$ \\ Paula Cordero-Pérez, ${ }^{3}$ Gabriela Alarcón-Galván, ${ }^{4}$ Liliana Torres-Gonzalez, ${ }^{3}$ \\ Linda E. Muñoz-Espinosa, ${ }^{3}$ and Nancy E. Fernández-Garza ${ }^{1}$ \\ ${ }^{1}$ Departamento de Fisiología, Facultad de Medicina, UANL, \\ Avenida Francisco I. Madero y Dr. Eduardo Aguirre Pequeño S/No, Col. Mitras Centro, \\ 64460 Monterrey, NL, Mexico \\ ${ }^{2}$ Departamento de Medicina Interna, Facultad de Medicina, \\ Universidad Autónoma de Nuevo León, 64460 Monterrey, NL, Mexico \\ ${ }^{3}$ Unidad de Hígado, Departamento de Medicina Interna, Facultad de Medicina, \\ Universidad Autónoma de Nuevo León, 64460 Monterrey, NL, Mexico \\ ${ }^{4}$ Servicio de Anatomía Patológica y Citopatología, \\ Hospital Universitario "José Eleuterio González," 64460 Monterrey, NL, Mexico
}

Received 27 August 2011; Accepted 12 October 2011

Academic Editor: Huaxin Sheng

Ischemia/reperfusion $(I / R)$ is a condition that stimulates an intense inflammatory response. No ideal treatment exists. Triflusal is an antiplatelet salicylate derivative with anti-inflammatory effects. S-adenosylmethionine is a metabolic precursor for glutathione, an endogenous antioxidant. Dextromethorphan is a low-affinity $\mathrm{N}$-methyl-D-aspartate receptor inhibitor. There is evidence that these agents modulate some of the pathways involved in I/R physiopathology. Intestinal I/R was induced in rats by clamping the superior mesenteric artery for 60 minutes, followed by 60 minutes of reperfusion. Rats either received saline or the drugs studied. At the end of the procedure, serum concentrations of tumor necrosis factor-alpha (TNF-alpha), malonaldehyde (MDA), and total antioxidant capacity (TAC) were determined and intestinal morphology analyzed. I/R resulted in tissue damage, serum TNF-alpha and MDA elevations, and depletion of TAC. All drugs showed tissue protection. Only triflusal reduced TNF-alpha levels. All drugs lowered MDA levels, but only triflusal and S-adenosylmethionine maintained the serum TAC.

KEYWORDS: Pharmacologic, preconditioning, Intestine, Injury, Rat, Inflammation 


\section{INTRODUCTION}

Intestinal ischemia occurs when there is an interruption of the blood supply to the intestine, or when there is inadequate organ perfusion due to conditions of shock or hypovolemia [1]. It is also an unavoidable condition during transplant and other surgical procedures, such as aortic repair. The initial damage caused by ischemia is further worsened by reperfusion. This entity is known as ischemia/reperfusion (I/R) injury. $\mathrm{I} / \mathrm{R}$ can adversely affect transplant outcomes and induces a systemic life-risking inflammatory response. $\mathrm{I} / \mathrm{R}$ injury is mediated by multiple mechanisms such as production of reactive oxygen species, intravascular thrombosis, inflammatory cell infiltration, and cytokine production [2]. Rodent models of intestinal I/R are useful in order to study the physiopathological mechanisms of I/R injury as well as in the search for much needed novel protective strategies [1]. In this study, we evaluated the value of triflusal (TRI), Sadenosylmethionine (SAME), and dextromethorphan (DEX) over intestinal I/R injury. All of these agents are currently in clinical use.

TRI is an antiplatelet drug related to but not derived from acetylsalicylic acid, that acts through the modulation of thromboxane synthesis but also possesses neuroprotective, antioxidant, and antiinflammatory properties [3]. TRI has been shown to decrease cerebral I/R injury and to prevent ischemiainduced cytokine expression in neural cells [4]. SAME is an amino acid that serves as a precursor to glutathione, a major physiological antioxidant, and serves an important function in opposing free radial production and oxidative stress generated by various pathological processes [5]. SAME has been shown to reduce brain and liver I/R injury, through decreased lipid peroxidation and induction of antioxidant defenses $[6,7]$. DEX is a antitussive and neuroprotective agent and a low-affinity N-methyl-D-aspartate (NMDA) receptor channel blocker. Studies have demonstrated that DEX protects the brain against I/R injury [8]. In one recent study, DEX was able to reduce intestinal I/R-induced lung injury in a rodent model [9]. To the best of our knowledge, the protective effects of these drugs against intestinal I/R injury have not been studied before.

\section{METHODS}

Animal procedures were performed in accordance with the proper use and care of laboratory animals, approved by the ethics committee of our institution. Experiments were performed on 25 male Wistar rats weighing 200-250 g. Animals were maintained under standard conditions, such as stable room temperature $\left(24 \pm 3^{\circ}\right)$, a $12 \mathrm{~h}$ light $/ 12 \mathrm{~h}$ dark cycle, and access to commercial rat pellets and water ad libitum. Briefly, after pentobarbital sodium anesthesia (Anestesal, Pfizer Inc, Mexico) (35 mg/kg, i.p), a midline laparotomy was performed. Animals were placed under heating lamp in order to preserve core body temperature at $\left(37^{\circ}\right)$. Ischemia was induced by cross-clamping the superior mesenteric artery for $60 \mathrm{~min}$ (ischemia), and removed to allow $60 \mathrm{~min}$ of reperfusion.

Drug dosages and time of administration were decided based on published literature and on doseresponse trials performed previously in our laboratory (unpublished data). Rats were randomly divided into five groups $(n=5)$ : (1) a sham-operated group (Sham), where laparotomy was performed but intestines were only manipulated but not made ischemic, (2) a control (CTL) group that was subjected to I/R as described above, (3) a TRI group, that received $100 \mathrm{mg} / \mathrm{kg}$ of TRI daily, for three days before I/R, (4) a SAME group, that received $100 \mathrm{mg} / \mathrm{kg}$ of SAME $1 \mathrm{~h}$ before surgery, and (5) a DEX group that received $100 \mathrm{mg} / \mathrm{kg}$ of DEX $1 \mathrm{~h}$ before surgery. All drugs were administered orally via gavage.

\subsection{Tissue Examination}

Immediately after concluding the reperfusion period, rats were sacrificed by exsanguination from the aorta and intestinal tissue samples were obtained. The samples were then fixed in $10 \%$ neutral buffered formalin overnight, embedded in paraffin, and $4 \mu \mathrm{m}$-thick sections were stained with hematoxylin and eosin $(\mathrm{H} / \mathrm{E})$ and examined under light microscope by a blinded pathologist. The Chiu score of mucosa injury was used to evaluate the degree of histological damage in the intestine, as detailed elsewhere [10]. The scale consists 
TABLE 1: Serum biochemical parameters: AST, LDH, and ALT levels.

\begin{tabular}{lccc}
\hline Group & AST $(\mathrm{U} / \mathrm{L})$ & LDH $(\mathrm{U} / \mathrm{L})$ & ALT $(\mathrm{U} / \mathrm{L})$ \\
\hline Sham & $145.25 \pm 12.1$ & $6080.75 \pm 1042.55$ & $52.5 \pm 11$ \\
CTL & $339.2 \pm 78.11^{*}$ & $10309.40 \pm 2240.16^{*}$ & $192 \pm 82.1^{*}$ \\
SAME & $214.25 \pm 11.47^{* *}$ & $10,200 \pm 1042.4 \mathrm{U} / \mathrm{L}$ & $74 \pm 16.9^{* *}$ \\
DEX & $236.75 \pm 16^{* *}$ & $7395 \pm 560^{* *}$ & $176 \pm 10.45$ \\
TRI & $166.75 \pm 65.9^{* *}$ & $3400 \pm 339^{* *}$ & $76 \pm 3^{* *}$ \\
\hline${ }^{*} P<0.05$ versus Sham $* * * 0.05$ verus $C T L$ & &
\end{tabular}

of values from of 0 to 5, where 0 normal mucosa; 1 development of subepithelial (Gruenhagen's) spaces; 2 extension of the subepithelial space with moderate epithelial lifting from the lamina propria; 3 extensive epithelial lifting with occasional denuded villi tips; 4 denuded villi with exposed lamina propria and dilated capillaries; 5 disintegration of the lamina propria, hemorrhage, and ulceration.

\subsection{Serum Analysis}

Blood samples were used to determine serum levels of AST (aspartate-aminotransferase), ALT (alanineaminotransferase), and LDH (lactate dehydrogenase) by standard biochemical automated methods, using commercially available kits and DT6011 and DTSC11 analyzers (System Vitros Chemical, Johnson and Johnson, USA). AST and LDH plasma levels serve as indirect markers of intestinal injury, while ALT is more specific for hepatic injury. Serum concentrations of TNF-alpha were determined using a rat ELISA kit (PeproTech, Mexico). Lipid peroxidation, expressed as malonaldehyde (MDA) levels, was assessed by the TBARS method using a commercially available colorimetric assay kit (Cayman chemicals, USA). Total serum antioxidant capacity (TAC) was assessed using a commercially available colorimetric assay kit (Cayman chemicals, USA). TAC values are expressed as Trolox Equivalents (TEs).

\subsection{Statistical Analysis}

SPSS 11.0 statistical software (SPSS Inc. Software, Chicago, Ill, USA) was used to analyze data using one-way analysis of variance (ANOVA) and with LSD post hoc test (when data were found to be normally distributed) and Kruskal-Wallis test (when data were not normally distributed) so as to determine comparison between groups and differences between groups, respectively. All values are expressed as mean $\pm \mathrm{SD}$, and $P<0.05$ was considered statistically significant.

\section{RESULTS}

\subsection{ALT, AST, and LDH}

AST values were significantly elevated after I/R in group CTL compared to sham group, and all treatment groups had AST levels significantly lower than CTL group (Table 1). LDH levels in group CTL were significantly increased compared to sham group. While groups TRI and DEX showed lower levels of LDH, levels in SAME group remained comparable to those in CTL group. After I/R, ALT values were increased in CTL group compared to sham group, but only TRI and SAME were able to reduce ALT elevations (Table 1). 


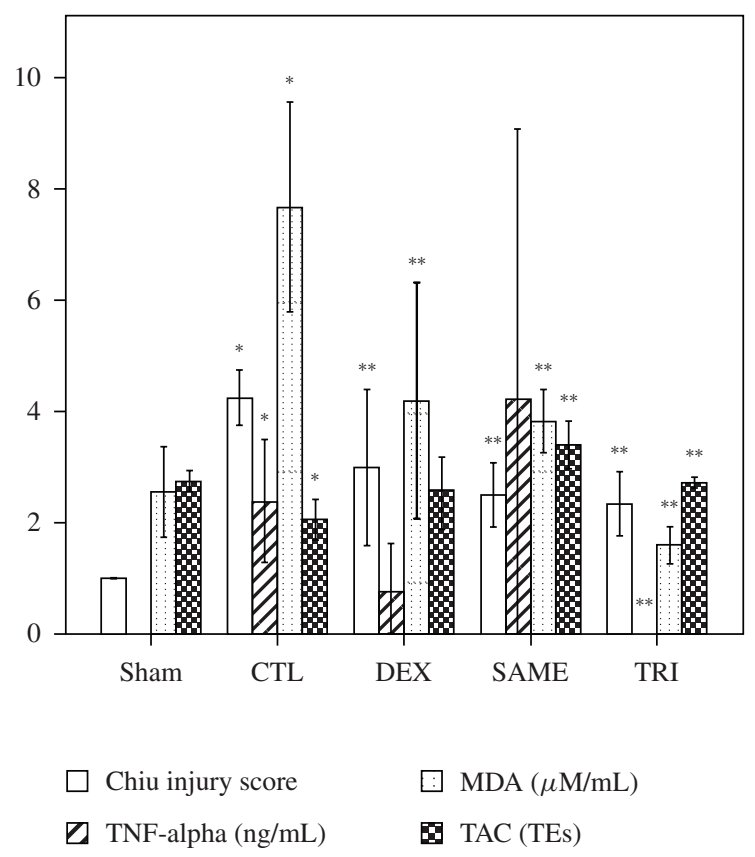

FIGURE 1: Chiu score and serum concentrations of TNF-alpha, MDA, and TAC. ${ }^{*} P<0.05$ versus Sham; ${ }^{* *} P<0.05$ versus CTL.

\subsection{Morphological Examination}

In sham group, Chiu score of mucosal injury was $1 \pm 0$, indicating negligible injury. In CTL group, severe mucosal injury was evident after I/R, reaching a Chiu score of $4.2 \pm 0.44$. In TRI group, there was some injury but it was significantly less than in CTL group (Chiu score: $2.7 \pm 0.95, P<0.05$, Figure 1 ). SAME group also showed reduced injury compared to CTL group, with Chiu score $2.5 \pm 0.57(P<0.05)$. DEX group showed reduced injury as well, reaching statistical significance at $3 \pm 1.41(P<0.05)$.

\subsection{Serum TNF-Alpha}

TNF-alpha levels remained undetectable in sham group. In CTL group, TNF-alpha levels reached $2.26 \pm$ $1 \mathrm{ng} / \mathrm{mL}$. TRI group had undetectable TNF-alpha levels by our assay as well, significantly lower than in CTL group $(P<0.05$, Figure 1$)$. The TNF-alpha levels in groups SAME and DEX were similar to those in CTL group (SAME: $4.2 \pm 4.9 \mathrm{ng} / \mathrm{mL}$; DEX: $0.74 \pm 0.88 \mathrm{ng} / \mathrm{mL}$ ).

\subsection{MDA and TAC}

Sham group had MDA concentrations of $2.56 \pm 0.829 \mu \mathrm{M} / \mathrm{mL}$. In CTL group, MDA levels were significantly increased $(7.42 \pm 1.72 \mu \mathrm{M} / \mathrm{mL}, P<0.05$, Figure 1$)$. All treatments were effective in reducing the elevations in serum concentrations of MDA (TRI: $1.59 \pm 0.27 \mathrm{U} / \mathrm{L} \mu \mathrm{M} / \mathrm{mL}$; SAME: $3.82 \pm 0.57 \mu \mathrm{M} / \mathrm{mL}$; DEX: $4.19 \pm 2.12 \mu \mathrm{M} / \mathrm{mL}, P<0.05)$, significantly lower to levels in CTL group. Sham group, serum TAC was $2.749 \pm 0.19$ TEs. In CTL group, serum antioxidants were significantly depleted $(2.05 \pm 0.36$ TEs, $P<0.05)$. In TRI group, serum TAC was preserved, with levels comparable to sham group and significantly higher than in CTL group ( $2.72 \pm 0.11$ TEs). In SAME group, serum TAC was actually increased compared to sham group $(3.39 \pm 0.43$ TEs, $P<0.05)$. However, in DEX group serum TAC showed only a trend towards higher levels when compared to CTL group $(2.58 \pm 0.6 \mathrm{TEs}, P=0.07)$. 


\section{DISCUSSION}

$\mathrm{I} / \mathrm{R}$ injury is a potentially devastating condition, associated with many surgical pathologies, and the search for therapeutic interventions is necessary, given that there is no standard treatment at present time. In this study, we evaluated the effects of three agents, with different mechanisms of action and clinical use, over intestinal I/R injury.

TRI was very effective in reducing intestinal I/R injury, as well as the associated TNF-alpha elevations, oxidative stress markers, and liver injury, as assessed by ALT elevations. TRI is an antiplatelet drug that inhibits both cyclooxygenase-1 (COX) and 2, decreasing prostaglandin and thromboxane production. Other COX-2 inhibitors have been also shown to protect the intestine against I/R injury, albeit mildly, while COX-1 inhibitors have little effects [11]. More studies would be required to show if TRI could be acting through a COX-2-dependent pathway. Platelet-endothelial interactions in the intestinal microvasculature play an important pathophysiological role in I/R injury [12]. Additionally, it has been demonstrated that TRI potently inhibits the transcription factors nuclear factor kappaB (NF-KappaB) and nuclear factor of activated $\mathrm{T}$ cells, responsible for the production of many inflammatory cytokines, such as TNF-alpha $[13,14]$, also essential in the pathophysiology of I/R injury. In experimental models of cerebral I/R injury, TRI is effective in reducing tissue injury, through antithrombotic, antioxidant, and antiinflammatory effects [3]. All these mechanisms could be responsible for the results we observed in this study.

Oxidative stress is an important mediator in pathological conditions of I/R injury, and antioxidant therapy has been widely studied as a protective strategy against this condition [15]. SAME is a precursor of glutathione, an antioxidant. Glutathione is a powerful cytoprotectant in the intestine, and glutathione supplementation has been proposed as a promising therapy for I/R injury [16]. In this study, SAME reduced intestinal I/R injury, the associated transaminase elevations, lipid peroxidation, and increased serum TAC. Studies involving I/R of organs such as the brain and liver have previously shown a protective effect of SAME, attributable to inhibition of lipid peroxidation and increased levels of protective antioxidants [6, 7]. However, SAME has also been effective in reducing inflammatory damage in a rodent model of colitis [17], and in vitro studies indicate that SAME is able to modulate inflammatory cytokine production, including TNF-alpha, in inflammatory cells [18]. However, we found no effects of SAME over TNF-alpha serum concentrations in our study, despite tissue protection. This dissociation between TNF-alpha production, oxidative stress, and tissue injury, after SAME treatment, was described in a recent study, involving a liver I/R model. Llacuna et al. [19] reported that after SAME treatment, ischemic liver cells had increased expression of TNF-alpha genes, despite increased antioxidant gene expression and protection against injury, possibly because differential effects of SAME over specific cellular populations. A similar effect could explain our results.

It is known that NMDA receptors exist in the enteric nervous system and NMDA antagonists are effective in reducing the functional alterations associated with intestinal I/R [20]. In previous studies, we have also demonstrated that ketamine, an NMDA antagonist, could reduce intestinal I/R injury, although whether this was due to NMDA-related effects, or to ketamine's antiinflammatory activity, remains unclear [10, 19, 21]. In the present study, we found that DEX also had mild protective effects. However, studies suggest that DEX not only acts through NMDA-inhibition, and other effects, such as calcium channel inhibition, modulation of c-fos expression, and possibly antiinflammatory effects, have been reported [2,9]. Reduced levels of TNF-alpha production, hepatoprotection, and inhibition of cytokine gene expression were observed after DEX administration in an endotoxemia model in rats [22], and DEX has also been demonstrated to inhibit macrophage free radical production in vitro [23]. In the present study, we found no effects of DEX treatment over either TNF-alpha levels or serum TAC, although we did find evidence of reduced lipid peroxidation. We also found no effects over ALT levels. The precise pathway involved in DEX's effects over I/R injury in nonnervous tissue warrants further research.

There are some limitations to this study. The drug doses used are higher than those that are clinically relevant. Furthermore, we studied acute injury and markers of inflammation and oxidative stress, but not outcome (such as mortality). We also did not study combination treatment, which we assume would be 
effective, since the drugs studied have different mechanisms of action. These issues require further investigation.

\section{CONCLUSIONS}

The search for pleiotropic drugs, capable of modulating the physiopathological mechanisms of I/R injury, to be used as protective therapies, is an active field of research. We have proposed such an application for three drugs currently in clinical use, each with markedly different mechanisms of action.

\section{CONFLICT OF INTERESTS}

The authors declare that they have no conflict of interests.

\section{ACKNOWLEDGMENTS}

The authors would like to thank the staff at their labs for invaluable assistance and MVZ Jose Luis Vazquez Juarez for providing laboratory animals and ensuring their care.

\section{REFERENCES}

[1] I. H. Mallick, W. Yang, M. C. Winslet, and A. M. Seifalian, "Ischemia-reperfusion injury of the intestine and protective strategies against injury," Digestive Diseases and Sciences, vol. 49, no. 9, pp. 1359-1377, 2004.

[2] S. E. Kong, L. R. Blennerhassett, K. A. Heel, R. D. Mccauley, and J. C. Hall, "Ischaemia-reperfusion injury to the intestine," Australian and New Zealand Journal of Surgery, vol. 68, no. 8, pp. 554-561, 1998.

[3] J. A. González-Correa and J. P. de la Cruz, "Triflusal: an antiplatelet drug with a neuroprotective effect?" Cardiovascular Drug Reviews, vol. 24, no. 1, pp. 11-24, 2006.

[4] S. N. Whitehead, N. A. Bayona, G. Cheng, G. V. Allen, V. C. Hachinski, and D. F. Cechetto, "Effects of triflusal and aspirin in a rat model of cerebral ischemia," Stroke, vol. 38, no. 2, pp. 381-387, 2007.

[5] C. S. Lieber, "S-adenosyl-L-methionine: its role in the treatment of liver disorders," American Journal of Clinical Nutrition, vol. 76, no. 5, pp. 1183S-1187S, 2002.

[6] M. A. Villalobos, J. P. de la Cruz, M. A. Cuerda, P. Ortiz, J. M. Smith-Agreda, and F. S. de la Cuesta, "Effect of S-adenosyl-L-methionine on rat brain oxidative stress damage in a combined model of permanent focal ischemia and global ischemia-reperfusion," Brain Research, vol. 883, no. 1, pp. 31-40, 2000.

[7] Y. B. Lee and S. M. Lee, "Effect of S-adenosylmethionine on hepatic injury from sequential cold and warm ischemia," Archives of Pharmacal Research, vol. 23, no. 5, pp. 495-500, 2000.

[8] P. M. Bokesch, J. E. Marchand, C. S. Connelly, W. H. Wurm, and R. M. Kream, "Dextromethorphan inhibits ischemia-induced c-fos expression and delayed neuronal death in hippocampal neurons," Anesthesiology, vol. 81, no. 2, pp. 470-477, 1994.

[9] R. Ben-Abraham, M. Guttman, R. Flaishon, N. Marouani, D. Niv, and A. A. Weinbroum, "Mesenteric artery clamping/unclamping-induced acute lung injury is attenuated by N-methyl-D-aspartate antagonist dextromethorphan," Lung, vol. 184, no. 6, pp. 309-317, 2006.

[10] C. R. Cámara, F. J. Guzmán, E. A. Barrera et al., "Ketamine anesthesia reduces intestinal ischemia/reperfusion injury in rats," World Journal of Gastroenterology, vol. 14, no. 33, pp. 5192-5196, 2008.

[11] T. V. Arumugam, N. Arnold, L. M. Proctor et al., "Comparative protection against rat intestinal reperfusion injury by a new inhibitor of sPLA2, COX-1 and COX-2 selective inhibitors, and an LTC4 receptor antagonist," British Journal of Pharmacology, vol. 140, no. 1, pp. 71-80, 2003.

[12] S. Massberg, G. Enders, R. Leiderer et al., "Platelet-endothelial cell interactions during ischemia/reperfusion: the role of P-selectin," Blood, vol. 92, no. 2, pp. 507-515, 1998.

[13] Y. Bayón, A. Alonso, and M. S. Crespo, "4-Trifluoromethyl derivatives of salicylate, triflusal and its main metabolite 2-hydroxy-4-trifluoromethylbenzoic acid, are potent inhibitors of nuclear factor $\kappa$ B activation," British Journal of Pharmacology, vol. 126, no. 6, pp. 1359-1366, 1999. 
[14] M. Aceves, A. Dueñas, C. Gómez, E. San Vicente, M. S. Crespo, and C. García-Rodríguez, "A new pharmacological effect of salicylates: inhibition of NFAT-dependent transcription," Journal of Immunology, vol. 173, no. 9, pp. 5721-5729, 2004.

[15] A. Ma, S. Qi, and H. Chen, "Antioxidant therapy for prevention of inflammation, ischemic reperfusion injuries and allograft rejection," Cardiovascular and Hematological Agents in Medicinal Chemistry, vol. 6, no. 1, pp. 20-43, 2008.

[16] H. Jefferies, J. Bot, J. Coster, A. Khalil, J. C. Hall, and R. D. McCauley, "The role of glutathione in intestinal dysfunction," Journal of Investigative Surgery, vol. 16, no. 6, pp. 315-323, 2003.

[17] H. S. Oz, T. S. Chen, C. J. McClain, and W. J. S. de Villiers, "Antioxidants as novel therapy in a murine model of colitis," Journal of Nutritional Biochemistry, vol. 16, no. 5, pp. 297-304, 2005.

[18] J. Yu, S. Sauter, and A. Parlesak, "Suppression of TNF- $\alpha$ production by S-adenosylmethionine in human mononuclear leukocytes is not mediated by polyamines," Biological Chemistry, vol. 387, no. 12, pp. 1619-1627, 2006.

[19] L. Llacuna, M. Marí, J. M. Lluis, C. García-Ruiz, J. C. Fernández-Checa, and A. Morales, "Reactive oxygen species mediate liver injury through parenchymal nuclear factor- $\kappa$ B inactivation in prolonged ischemia/reperfusion," American Journal of Pathology, vol. 174, no. 5, pp. 1776-1785, 2009.

[20] C. R. Cámara-Lemarroy, F. J. Guzmán-de la Garza, G. Alarcón-Galván, P. Cordero-Pérez, and N. E. FernándezGarza, "The effects of NMDA receptor antagonists over intestinal ischemia/reperfusion injury in rats," European Journal of Pharmacology, vol. 621, no. 1-3, pp. 78-85, 2009.

[21] F. J. G. Garza, C. R. Cámara-Lemarroy, R. G. Ballesteros-Elizondo, G. Alarcón-Galván, P. Cordero-Pérez, and N. E. Fernández-Garza, "Ketamine reduces intestinal injury and inflammatory cell infiltration after ischemia/reperfusion in rats," Surgery Today, vol. 40, no. 11, pp. 1055-1062, 2010.

[22] G. Li, Y. Liu, N. S. Tzeng et al., "Protective effect of dextromethorphan against endotoxic shock in mice," Biochemical Pharmacology, vol. 69, no. 2, pp. 233-240, 2005.

[23] S. L. Liu, Y. H. Li, G. Y. Shi et al., "Dextromethorphan reduces oxidative stress and inhibits atherosclerosis and neointima formation in mice," Cardiovascular Research, vol. 82, no. 1, pp. 161-169, 2009.

\section{This article should be cited as follows:}

Carlos R. Cámara-Lemarroy, Francisco J. Guzmán-de la Garza, Paula Cordero-Pérez, Gabriela AlarcónGalván, Liliana Torres-Gonzalez, Linda E. Muñoz-Espinosa, and Nancy E. Fernández-Garza, "Comparative Effects of Triflusal, S-Adenosylmethionine, and Dextromethorphan over Intestinal Ischemia/Reperfusion Injury," TheScientific WorldJOURNAL, vol. 11, pp. 1886-1892, 2011. 

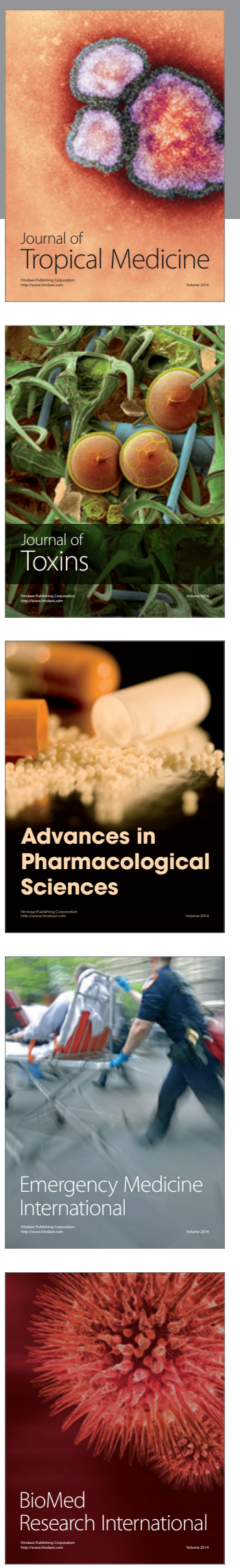
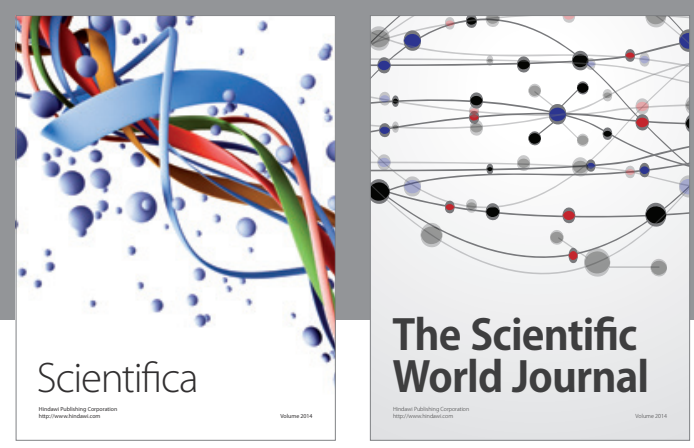

The Scientific World Journal
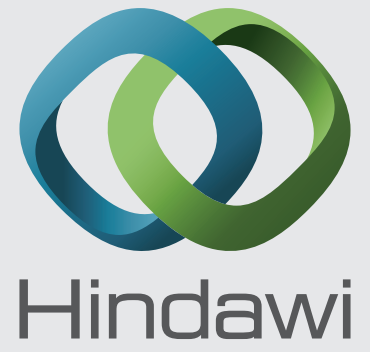

Submit your manuscripts at

http://www.hindawi.com
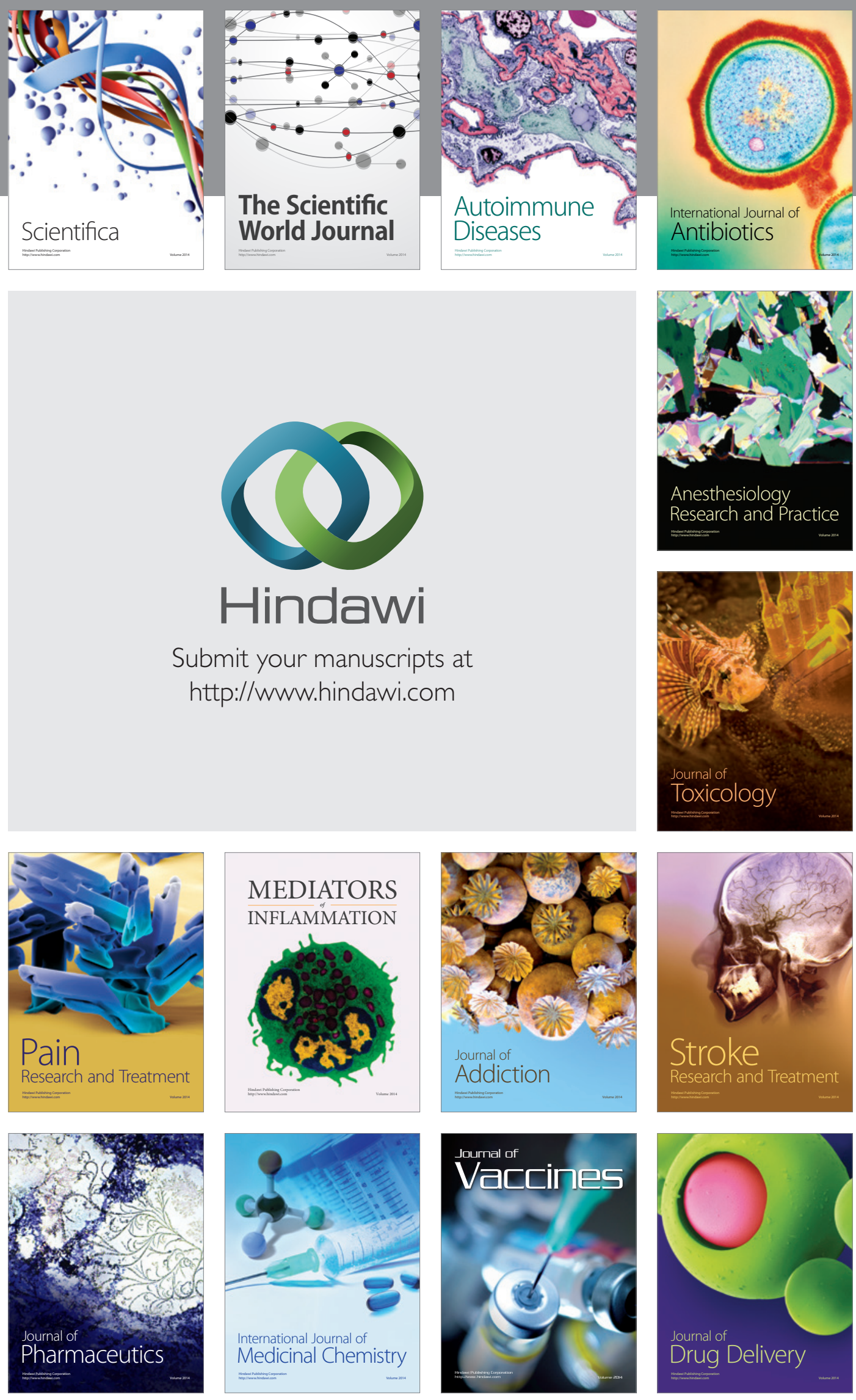